\title{
Macroscopic and microscopic mechanical behaviors of climbing tendrils
}

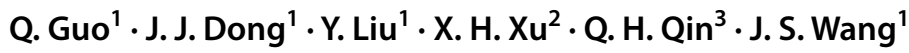

Received: 3 December 2018 / Revised: 4 January 2019 / Accepted: 23 January 2019 / Published online: 28 March 2019

(C) The Chinese Society of Theoretical and Applied Mechanics and Springer-Verlag GmbH Germany, part of Springer Nature 2019

\begin{abstract}
Tendril-bearing climbing plants must recur to the tendril helices with chiral perversion or dual chirality for climbing and to obtain sun exposure. Despite researchers' prolonged fascination with climbing tendrils since Darwin's time and even earlier, why the soft and slender tendrils can bear heavy loads such as the self-weight of a plant or additional load caused by rain remains elusive. In this paper, we take towel gourd tendrils as an example and investigate the macroscopic and microscopic mechanical behaviors of tendrils through experiments and simulations. Our study indicates that the tendril filament exhibits rubber-like hyperelastic behaviors and can particularly endure large elongation, which is mainly attributed to the superelasticity of the cellulose fibril helix contained in the cell wall. Combination of the tendril helical structure with dual chirality or chiral perversion at a macroscale and a cellulose filament helix at a subcellular level creates superior elasticity for biological species relying on support and climbing. This study provides deep insight into the structure-property relationship of climbing tendrils, and the relationship is useful for the bioinspired design of composite systems with superior elasticity.
\end{abstract}

Keywords Climbing tendril $\cdot$ Mechanical behaviors $\cdot$ Structure-property relationship $\cdot$ Large elongation

\section{Introduction}

Over billions of years of evolution, a wide range of high-performance biological materials in nature have been developed using conventional and economic materials in an extremely efficient and economical way [1]. Their well-known representatives are bone [2], nacre [3, 4], wood, anchor spicule of Euplectella aspergillum [5], spider web [6], and luffa sponge [7], just to name a few. These natural materials usually integrate adaptive growth, remodeling and optimized hierarchical structures at multiple length scales to achieve outstanding properties such as high toughness and superior elasticity so as to fulfill some specific biological functions

\section{J. S. Wang}

wangjs@tju.edu.cn

$1 \quad$ Tianjin Key Laboratory of Modern Engineering Mechanics, Department of Mechanics, Tianjin University, Tianjin 300054, China

2 State Key Laboratory of Nonlinear Mechanics (LNM), Institute of Mechanics, Chinese Academy of Science, Beijing 100080, China

3 Research School of Engineering, Australian National University, Canberra, ACT 2601, Australia
$[8,9]$. Their underlying principles and mechanisms may serve as a source of inspiration to guide the design of materials with novel functions. For instance, the flaw tolerance [2], gecko-inspired reversible adhesive and super-hydrophobicity of lotus leaves [10] have been successfully translated to the material world. For such translations, understanding and revealing the structure-property relationship in biological materials is a fundamental and promising scientific and technological challenge in both biology and material science.

Tendrils, the long, slender and filamentous organs derived from stems, leaves or flowers of climbing plants are typical natural fiber composites [11-13]. The main chemical components of the tendrils are cellulose, hemicellulose and lignin. According to the climbing modes, climbing plants were roughly catalogued by Darwin in five classes, i.e., twining plants, leaf-climbers, tendril-bearers, root-climbers and hook-climbers [12]. Different to other climbing plants, the tendril-bearers such as towel gourd, cucumber and cucurbit must recur to the tendril filament helices for grasping the supports in order to grow vertically and compete for sunlight $[13,14]$. During their growth, tendril-bearers generally let the straight or bended tendril filaments circumnutate like a twining plant, but in a faster way to find supports. Just as Darwin commented "It first places its tendrils ready for 
action, as a polypus places its tentacula" $[12,15]$. Once the tendril filaments touch and wind around the anchored support, they always coil themselves to form helical structures with opposite handedness, which is called chirality perversion, as shown in Fig. $1[13,14,16]$. Otherwise, they only form a free coil with left- or right-handedness. Such helical structures with dual chirality act as an elastic spring-like connection between the plant and anchored support, providing the plant with the flexibility and resistance to high wind and self-weight.

Climbing tendrils have continuously fascinated numerous researchers from biology, mechanics, material science and engineering areas since Darwin's time and even earlier [12]. Modeling the tendrils as thin elastic rods with intrinsic curvature, Goriely and Tabor's work shows that formation of the tendril perversion can be explained in terms of a dynamical analysis of the solutions to the Kirchhoff equations for thin elastic rods [14]. The tendril perversion phenomena were also elucidated as a result from the topological conservation law [16]. It is shown that cucumber tendril coiling can be attributed to the asymmetric contraction of an internal fiber ribbon of specialized cells [11]. The tendril can overwind during extension and exhibit an initially soft response and then strong strain-stiffening at large extensions, induced by the combination of mechanical asymmetry, helical perversions and large ratios of bending to twisting stiffness [11]. Recently, Wang et al. [13] showed through experiments and theoretical modelling that the towel gourd tendrils have hierarchical chiral structures, and the change in the helical angle of cellulose fibrils at the subcellular level induces

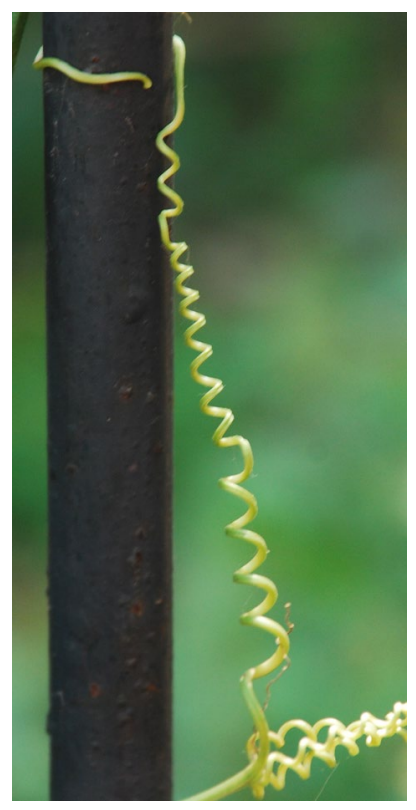

Fig. 1 Tendril helix of towel gourd with chirality perversion or dual chirality an intrinsic torsion of tendrils, leading to the formation of the helical morphology of tendril filaments. Furthermore, it has been revealed that when the towel gourd tendril helix is tensioned, the interactions between macroscopic helical structure and microscopic cellulose helices can induce an initial overwinding and a subsequent unwinding, demonstrating a nonlinear stretch-twist coupling behavior [17]. Despite the prolonged fascination with climbing tendrils, we know surprisingly little about their structure-property relationship. Open questions are numerous. For example, why such slender and flexible helical structures can bear the heavy wind load and the weight of plant and how the microstructures affect the properties and function of tendrils are still open questions.

On the other hand, considerable research has been inspired to mimic the properties and functional aspects of climbing tendrils due to their intriguing chiral shape and adaptive deformation in response to external stimuli $[18,19]$. For example, a cylindrical tube with chiral architected that can convert axial compression loads into angular rotation deformation has been designed by imitating the deformation and movement of tendril helices with chirality perversion [20]. Currently, some biomimetic materials and devices, including responsive materials, artificial muscles and torsional actuators in response to specific external stimuli such as temperature and humidity, have been created through the helical assembly of synthetic polymers, nanowires and carbon nanotubes. For instance, actuating fibers that respond to solvent and vapor stimuli have been recently designed and produced through the hierarchical helical assembly of aligned carbon nanotubes, inspired by the chiral microstructures of tendrils at multiple structural levels [21, 22]. A biomimetic conductive tendril created by judiciously incorporating helical conductive yarns into an elastic polymer-based coiled structure has been designed for ultrastretchable and integratable electronics, muscles and sensors [23]. However, most of the current research efforts on biomimetic materials and devices mainly focus on simply mimicking some specific properties and functions of climbing tendrils. To further improve their performance and create materials with desired mechanical properties such as superior elasticity, high strength and adaptive deformation, a thorough understanding of the structure-property relationship of climbing tendrils is extremely critical.

Herein, taking the towel gourd tendril as an example, we focus on how the hierarchically chiral structures contribute to the mechanical properties and load-bearing capacity of tendril helices. We conduct tension experiments and finite element method (FEM) simulation of tendril helices to investigate the macroscopic and microscopic mechanical behaviors of tendrils. The Gent hyperelastic material model is used to describe the tensile behaviors of tendril filaments under large elongation. The microscopic mechanism 
underlying the mechanical behaviors of tendril filaments at large elongation is revealed. We show that the superior loadbearing capacity of tendril helices is mainly attributed to the combination of tendril helical structures at a macroscale and the cellulose filament helices at a subcellular level.

\section{Hierarchically chiral structures of tendril helices}

Through scanning electron microscopy (SEM), it is observed that the approximately circular cross section of the tendril filament mainly consists of a surface layer, an inner layer and a core as shown in Fig. 2 [13]. The surface layer (L1) is composed of epidermis cells, the inner layer (L2) consists of primarily parallelized cells, which contain cellulose fibril helices with left handedness in cell walls, and the core (L3) consists of relatively large, soft and hollow tube-like cells [13]. Each cell in the inner layer can be taken as a chiral rod or beam [24, 25], and thus the tendril filament can be viewed roughly as a chiral fiber bundle. At macroscale, the towel gourd tendrils usually form helical structures with opposite handedness. The diameter of the tendril's helical structures generally ranges from $4 \mathrm{~mm}$ to $6 \mathrm{~mm}$, and the length of the helical axis is about $3-10 \mathrm{~cm}$. The diameter of tendril filaments ranges from approximately $1 \mathrm{~mm}$ to $3 \mathrm{~mm}$. Thus, the towel gourd tendril helices have typical hierarchically chiral structures sweeping from a subcellular level to the macroscale. In the following section, we investigate how these chiral structures at different levels affect the mechanical behaviors of tendril helices.

\section{Mechanical behaviors of tendril helical structures with dual chirality}

Two or more tendril helices with opposite handedness are joined together by approximately straight tendril filament parts, forming helical structures with dual chirality or chiral perversion. Such tendril helical structures provide a flexible and elastic connection between the plant stem and support object. To investigate the mechanical behaviors of towel gourd tendril coils, tensile experiments were conducted on the mechanical behavior of the tendril coils. The mature tendril helical structures with three left- and right-handed coils were taken as samples. The tendril samples with axial lengths ranging from $23 \mathrm{~mm}$ to $28 \mathrm{~mm}$ were quasi-statically tensioned till fracturing using in situ tension and compression testing machine. The two ends of tendril filaments were fixed. Figure 3 shows that the tendril helical structures quickly stiffen at large elongation. During the tension experiments, the axial displacements at which the tendril helical filaments break reached about $30 \mathrm{~mm}$. Furthermore, these tendril helices can usually restore its initial shape after unloading at relatively large axial displacements such as $26 \mathrm{~mm}$ or $27 \mathrm{~mm}$, suggesting that they possess superelasticity. In addition, during the extension period, the helical pitch and coil number become increased, i.e., the tendril helical structures exhibit obviously overwinding rather than

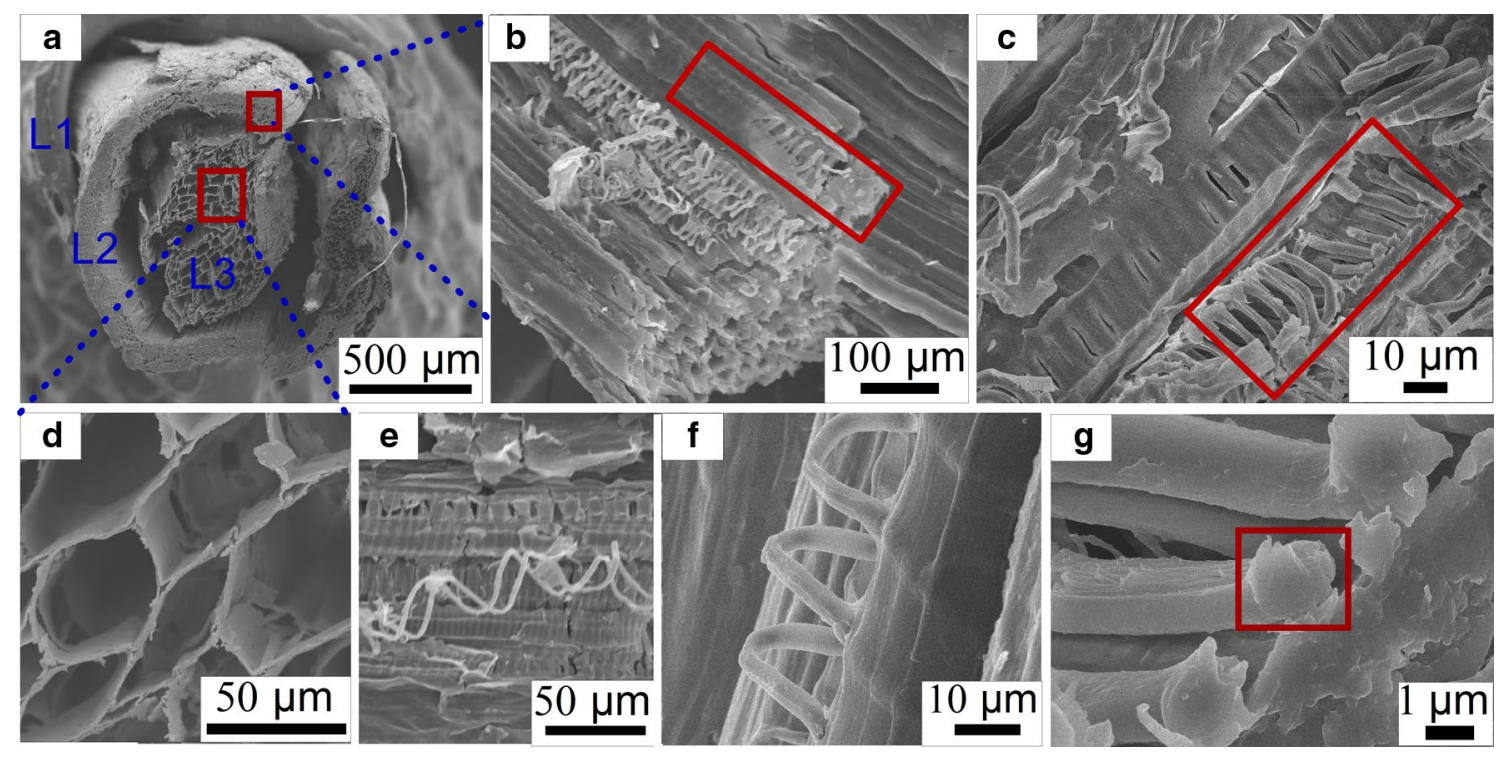

Fig. 2 Hierarchical structures of tendril filaments. a Cross section of a tendril filament; b inner layer of a tendril filament dissected longitudinally; $\mathbf{c}$ cells in the straight part of a tendril filament connecting the left- and right-handed parts; $\mathbf{d}$ core layer of a tendril filament; $\mathbf{e}$ two cellulose fibril helices separated from the cell wall; $\mathbf{f}$ a single cellulose helix; $\mathbf{g}$ cross section of a cellulose fibril. (figure reproduced from Ref. [14]) 


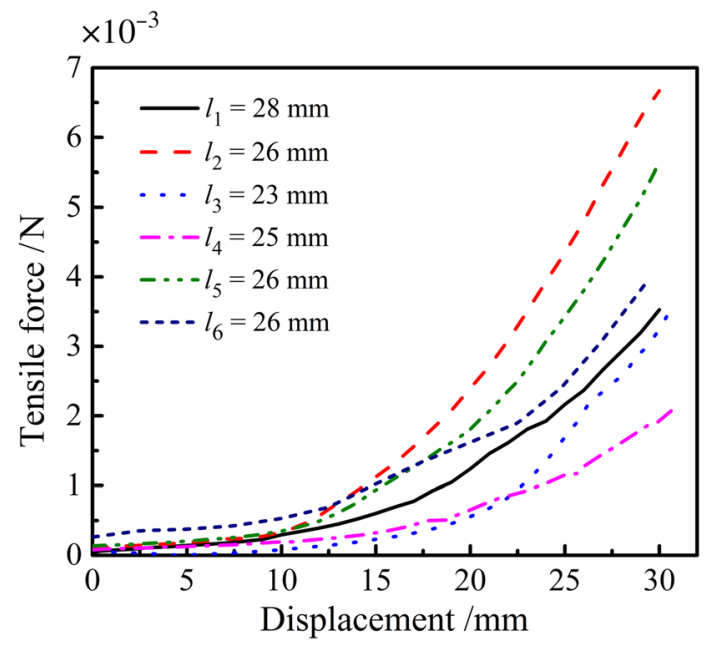

Fig. 3 Force-displacement curves of towel gourd tendrils

unwinding. As shown in Fig. 4, the coil number of a mature tendril helical structure with left- or right-handedness increased from 10 to 11, whereas for a young tendril, the coil number reduced from 4 to 3 . Such overwinding and unwinding phenomena of helical structures with chirality perversion or dual chirality are similar to those of cucumber tendril coils under tension [11]. It is found that the ratio of bending rigidity $B$ of tendril filament to twisting rigidity $C$ controls overwinding or unwinding during stretching of the tendril helix [11]. For a mature towel gourd tendril, the bending rigidity $B$ is always larger than the twisting rigidity, i.e. $B / C>1$. In this case, the chirality perversion usually increases the tension force and the tendril filament overwinds with the increasing of number of turns. Furthermore, our previous work [17] showed that the interactions between macroscopic and microscopic chiral structures can also induce nonlinear stretch-twist coupling behaviors.

When subjected to loads from strong wind and rain or the self-weight of the plant, the tendril helical structures with dual chirality or chirality perversion can be extended largely in the axial direction, simultaneously accompanied by their rotation motions. Such coupling between linear and rotation motions are induced by the chiral perversion, which plays an important role to shape the mechanical behaviors of tendril helices. To investigate further the effects of chiral perversion, we simulated the tension behavior of tendril helical structures for one helix, and two and three helices, as shown in Fig. 5, using ABAQUS FEM software [26]. One end of the tendril filament usually winds tightly on support objects, and another connects to the plant. Correspondingly, in the simulation, one end of tendril filament was fixed and another was loaded by a uniaxial tension force $F$ denoting the wind and rain loads or the self-weight of the plant. Based on the geometrical measurements of the tendril shown in Fig. 2, the helical radius was taken as $2.5 \mathrm{~mm}$, and the radius of the tendril filament cross section is $1 \mathrm{~mm}$. The tendril cell has chiral elastic properties due to the helical winding of cellulose fibrils in the cell wall. For convenience, we treated the tendril filament as an elastic rod rather than a chiral rod in order to focus our attention mainly on the effect of chirality perversion. According to their mechanical responses, the elastic modulus $E$ and Poisson's ratio $v$ of the tendril filament were reasonably taken as $5 \mathrm{GPa}$ and 0.33 , respectively. The C3D8R element [26] was adopted for the tendril

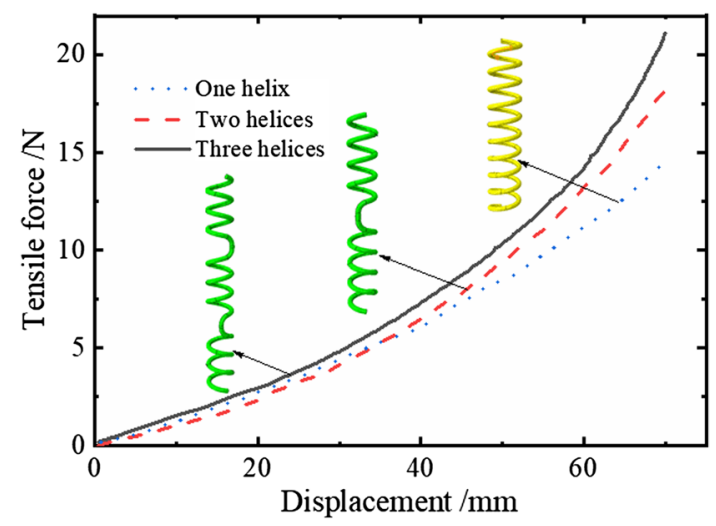

Fig. 5 Force-displacement curves of helical structures with one helix, two helices and three helices a

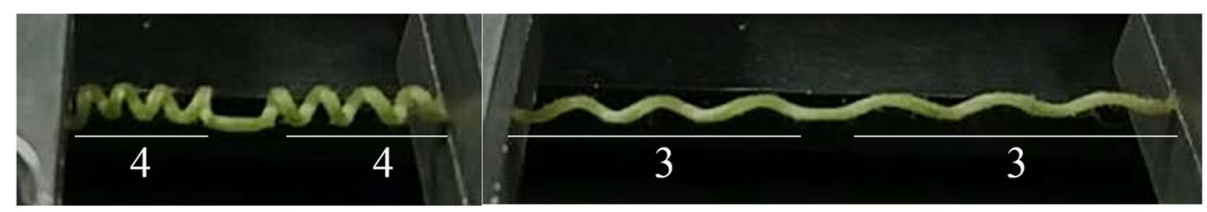

b

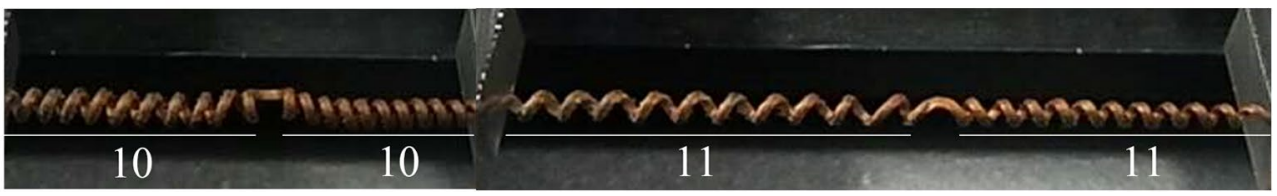

Fig. 4 Unwinding and overwinding behaviors of stretched towel gourd tendrils. a Young towel gourd tendril. b Mature towel gourd tendril 
filament and 8000 are meshed. To show the effects of the chirality perversion, the tendril filament lengths of helical structures are kept unchanged in different cases of chirality. The length of the center line of helical structures was taken as about $7 \mathrm{~mm}$. Figure 5 shows that the tension force of the tendril helical structure increases linearly first and then nonlinearly with the displacement, i.e., the helical structure quickly stiffens. This is because when tensioned, deformations of the tendril helical structure are mainly in the form of bending and twisting. With the increase of tension force, the axial extension deformation becomes prominent in the stage of large extension with the displacement ranging from $50 \mathrm{~mm}$ to $70 \mathrm{~mm}$. The forces in the cases of two and three helices are obviously larger than that of one helix, suggesting that the chirality perversion or dual chirality can effectively improve the elasticity of the tendril helical structure through the coupled linear and rotation motions. It should be mentioned that similar effects of chirality perversion on the tension behaviors of tendril helical structures can be obtained with other values of elastic modulus and Poisson's ratio.

\section{Mechanical behaviors of tendril filaments}

The elasticity of helical structures plays a significant role in the biological function of tendrils, such as climbing. When subjected to small loads, the tendril's helical structures undergo small elongation only, and the elastic deformation mainly comes from the changes of geometrical curvature and torsion of the tendril filament. When subjected to large loads, the tendril helical structures often undergo large elongation, and the axial extension deformation of the tendril filament becomes prominent. For example, when the tendril helix is stretched from a helical shape to a nearly straight line, the axial strain of a tendril filament can reach about $6 \%$ or even larger. In some cases, with extreme loading conditions, such as strong winds and heavy rains, the tendril helix can be suddenly pulled into a straight line and even stretched further. During this loading process, the maximal axial strain of the tendril filament can be even larger than $10 \%$. And then the tendril helix tends to restore its original shape after unloading. Such superior elasticity requires that the tendril helix not only has a periodic and helical structure with dual chirality, but also that the tendril filament has the ability to endure large elongation. Furthermore, the latter determines in a large degree the load-bearing capacity of tendril helical structures.

To investigate the mechanical behavior of tendril filaments under large elongation, we conducted tension experiments. The tendril filament samples taken from mature tendril helical structures generally have intrinsic torsion, and they are made to be straight after being twisted in a reverse direction. These straight tendril filament samples have a unified length of $30 \mathrm{~mm}$, and are quasi-statically tensioned till fracturing using an in situ tension testing machine. Both ends of the tendril filament are fixed. The obtained force-displacement curves of six tendril filament samples are given in Fig. 6. It shows that the tensile force increases nonlinearly with the increase of displacement. The breaking stain at which the tendril filament is fractured reaches about $46 \%$, which indicates the strong ability of tendril filament to endure large elongation.

Here, we use the Gent hyperelastic material model [27] to describe the extension behavior of tendril filaments undergoing large deformation. The model is mainly used to phenomenologically model the rubber elasticity induced by the limiting chain extensibility. The strain energy density function for the Gent model [27] is

$W=-\frac{\mu J_{m}}{2} \ln \left(1-\frac{I_{1}-3}{J_{m}}\right)$,

where $\mu$ and $I_{1}$ are the shear modulus and the first invariant of the left Cauchy-Green deformation tensor, respectively, and $J_{m}=I_{m}-3$ with $J_{\mathrm{m}} \gg 1$.

The Cauchy stress is given by

$\boldsymbol{\sigma}=-p \boldsymbol{I}+\frac{\mu J_{m}}{J_{m}-I_{1}+3} \boldsymbol{B}$,

where $\boldsymbol{I}$ and $\boldsymbol{B}$ are the identity tensor and the left CauchyGreen deformation tensor, respectively. $\boldsymbol{B}$ can be expressed as

$B=\lambda^{2} \boldsymbol{n}_{1} \otimes \boldsymbol{n}_{1}+\frac{1}{\lambda}\left(\boldsymbol{n}_{2} \otimes \boldsymbol{n}_{2}+\boldsymbol{n}_{2} \otimes \boldsymbol{n}_{2}\right)$,

where $\boldsymbol{n}_{i}(i=1-3)$ are the principal directions.

For uniaxial extension of a tendril filament in an axial direction, the principal stretches are $\lambda_{1}=\lambda$ and $\lambda_{2}=\lambda_{3}$.

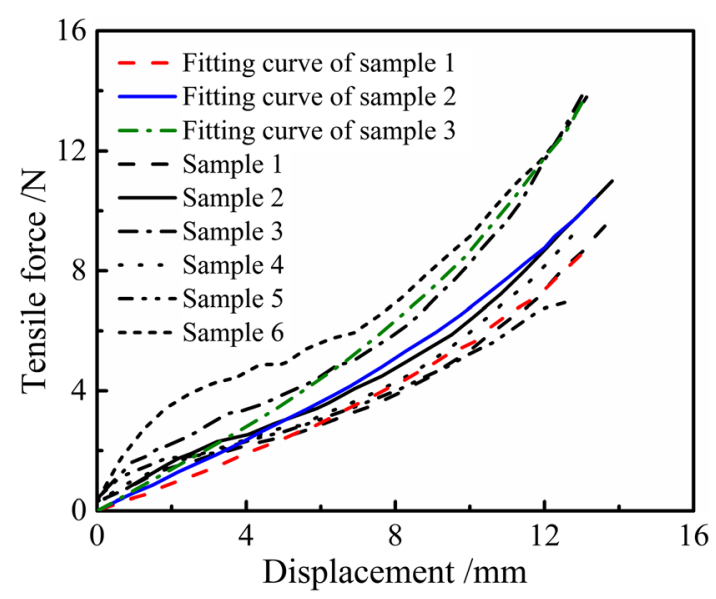

Fig. 6 Force-displacement curves of towel gourd tendril filament 
According to the incompressible condition $\lambda_{1} \lambda_{2} \lambda_{3}=1$, we can have $\lambda_{2}=\lambda_{3}=\frac{1}{\sqrt{\lambda}}$. Then we obtain

$\sigma_{11}=\frac{\mu J_{m}\left(\lambda^{3}-1\right)}{\lambda\left(J_{m}-I_{1}+3\right)}, \sigma_{22}=\sigma_{33}=0$

Considering the engineering strain $\varepsilon=\lambda-1=\frac{u}{L}$, the relation between the tensile force $F$ and displacement of the tendril filament under large elongation can be given as

$F=\frac{\pi r^{2} \mu J_{m}}{J_{m}-I_{1}+3}\left[\left(\frac{u}{L}\right)^{2}-\frac{L}{u}\right]$

Here, $u$ and $L$ are the extension displacement and the original length of the tendril filament, respectively. $r$ is the tendril filament's cross section radius, and the measured value of $r$ is $1 \mathrm{~mm}$.

According to Eq. (5), the force-displacement curves of tendril filaments obtained from the tensile experiments are fitted. As shown in Fig. 6, the fitting curves agree well with the experimental curves. For simplicity, only three curves are fitted. It should be emphasized that there are only two unknown material parameters, i.e., the shear modulus $\mu$ and the parameter $J_{m}$ in Eq. (5). The values of $\mu$ and $J_{m}$ can be drawn directly from the fitting curves, and they are $\mu=0.941$ $\mathrm{GPa}, J_{m}=1.355$ for sample $1, \mu=0.941 \mathrm{GPa}, J_{m}=2.0$ for sample 2 , and $\mu=0.6435 \mathrm{GPa}, J_{m}=1.653$ for sample 3 . It is demonstrated that the tendril filament obviously possesses rubber-like elasticity, and the Gent model can effectively capture the tensile behaviors of the tendril filament undergoing large deformation. It should be mentioned that the freedom at the two ends of the tendril filament is limited, which constrains the effects of twisting. Thus, for simplicity, the effects of intrinsic twisting on the tensile behaviors produced during growth are not considered here.

\section{Mechanical behaviors of the tendril cell}

Cells are basic structural elements which determine in a large degree the mechanical behaviors and elastic properties of tendril filaments. To uncover the microscopic mechanism of large deformation of a tendril filament, the mechanical behaviors of the tendril cell are herein investigated using FEM simulation. As mentioned above, the tendril helical structures have hierarchically chiral structures. At a cellular level, the tendril cell can be viewed as a chiral rod. Different to wood cells, the soft and thin tube-like tendril cell wall contains helical cellulose fibrils with a cross-section diameter of 1-2 $\mu \mathrm{m}$. The distinct changes of helical shapes of a tendril filament during swelling and deswelling experiments suggest that the cellulose fibrils are mainly responsible for the deformation of the tendril cell [13]. As mentioned above, the tendril filaments can be viewed as a cell bundle, in which the cells are bonded together. Therefore, the cellulose fibrils at a subcellular level play a significant role in the macroscopic mechanical properties of tendril filaments. The tendril cell wall is mainly composed of hemicellulose, cellulose and lignin, and usually acts as a typical incompressible hyperelastic material. It is suggested that the tensile behavior of a cell wall under large elongation can be described here by the strain energy density function for the incompressible Mooney-Rivlin constitutive relation [28]

$W=C_{1}\left(I_{1}-3\right)+C_{2}\left(I_{2}-3\right)$,

where $W$ is the strain energy density function, and $C_{1}$ and $C_{2}$ are the Mooney constants.

For the cellulose fibrils inside the tendril filament, previous studies on nanowires and carbon nanotubes indicated that the helix of a thin and slender filament possesses superelasticity; i.e., the helix can endure a large elongation and recover to its original shape after unloading. Due to their thin and slender geometrical shape and periodic helical structure, only small axial deformations occur for the cellulose fibrils when the tendril filaments undergo large elongation. Therefore, the cellulose fibrils are taken as linearly elastic rods with isotropic elastic properties during the large elongation of tendril filaments. Considering its microstructure and material properties, the tendril cell is modeled as a simple tube-helix structural element, as shown in Fig. 7, in which the tube made of thin and relatively soft hyperelastic material contains closely the helix with relatively stiff cellulose fibrils. Up to now, the measured values of elastic property parameters of tendril cell walls and cellulose fibrils have not been available. The tube-helix structural model here is intended to be used to investigate the basic mechanical features of the tendril cell in order to reveal the microscopic mechanism responsible for the rubber-like elasticity of the tendril filament. Thus, the predicted values of elastic parameters are adopted for simplicity. Here, the values of $C_{1}$ and $C_{2}$ of the tube were roughly predicted as $C_{1}=0.965$ and $C_{2}=0.2412$ based on its soft nature. The axial length of the tube and helix was taken as $51 \mu \mathrm{m}$, and the outer and inner diameters were $4.75 \mu \mathrm{m}$ and $4.5 \mu \mathrm{m}$, respectively. The C3D8R and C3D10M elements [26] were adopted for the cellular fibril and cell wall, respectively. Their numbers were 2100 and 2300, respectively. The Poisson's ratio of the cellulose fibril was taken as 0.4. Because of its hyperelasticity, the cell wall can be generally subjected to a strain larger than $40 \%$. Due to its periodic and helical structures, the cellulose fibril helix can usually endure a large elongation during stretching, providing a compatible deform between the cell wall and cellulose fibril helix. Thus, although the interaction between the cell wall and cellular fibril may be far more 


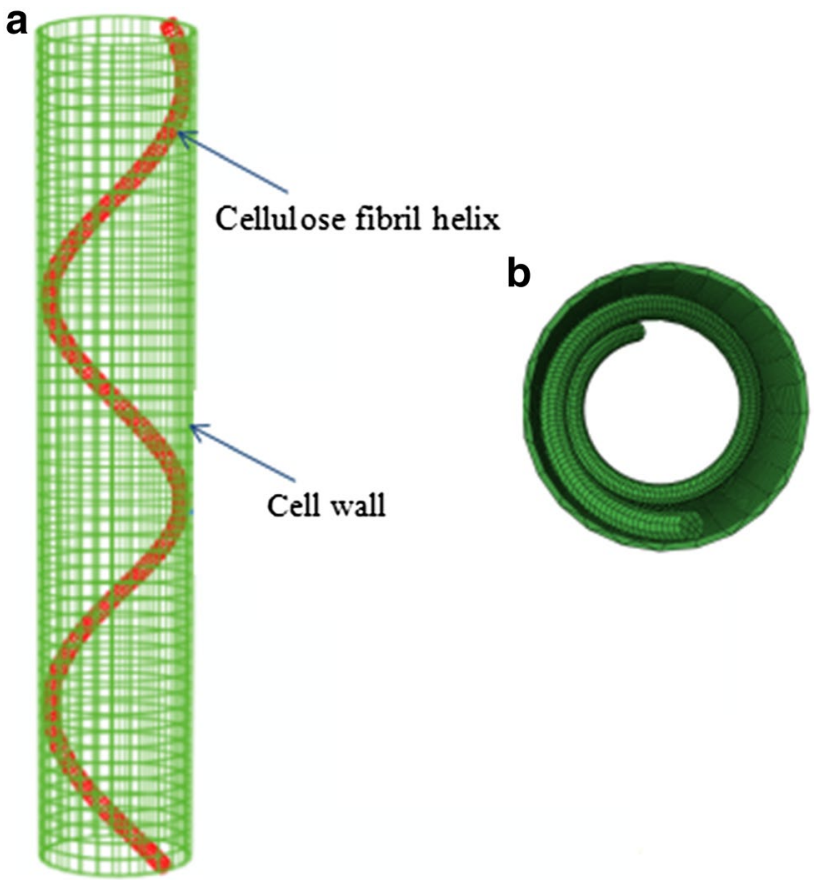

Fig. 7 a Side, and $\mathbf{b}$ top views of the tube-helix structural element

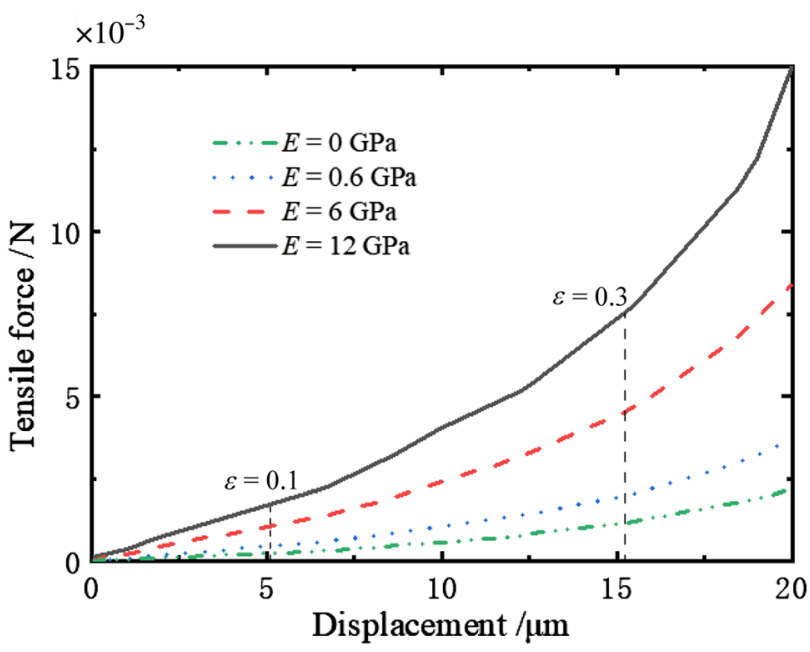

Fig. 8 Force-displacement curves of a tube-helix structure for different elastic modulus

complex, it was simply considered that the cell wall and the cellular fibril are fully bonded.

The axial tensile forces are applied at the tube ends. The tube ends can only deform in an axial direction and other freedoms are constrained. In Fig. 8, the force-displacement curves of the tube-helix structure are plotted for different elastic moduli of the cellulose fibril. The original helical angle and radius are taken as $80^{\circ}$ and $4 \mu \mathrm{m}$, respectively.
The cross-section diameter is $1 \mu \mathrm{m}$. The displacement ranges from 0 to $20 \mu \mathrm{m}$, corresponding to the maximal axial strain of $40 \%$. It shows that the tensile force increases nonlinearly with the displacement or deformation. Generally, a typical force-displacement curve can be divided into three stages, the linear stage, the nonlinear stage and the large elongation stage. In the linear stage, the axial strain is smaller than $10 \%$; the elasticity of the contained helix mainly comes from the bending and twisting deformations of the cellulose fibril, and the axial deformation of the cellulose fibril is negligible, leading to a distinctly linear tensile force-displacement relationship. Meanwhile, in the nonlinear stage, the axial strain varies from $10 \%$ to $30 \%$, and the elasticity of the contained helix mainly derives from the combination of bending, twisting and axial extension deformations, inducing the nonlinear force-displacement relationship. In the large elongation stage the axial strain is larger than $30 \%$, the contained helix is stretched into a nearly straight configuration, and the elasticity of helix is dominated by the axial deformation of the cellulose fibril. As shown in Fig. 8, the elastic modulus of the helical fibril affects significantly the force-displacement relationships of the tube-helix structure. It is shown that to produce a similar displacement, the force applied to the tubes with the contained helix is much larger than that that without the helix (i.e., $E=0$ ). Furthermore, the relatively stiff fibril can effectively improve the elasticity of the tubehelix structure, especially in the nonlinear stage and large elongation stage. This is because during the large elongation of tendril filament, the axial deformation of the helical fibril becomes prominent, leading to a large tensile force. Figure 8 indicates that the elasticity of the tendril filament under large elongation mainly derives from the contained helix. As mentioned above, the tendril filament can be taken as a cell bundle in which the cells are bonded together. The forcedisplacement curves of the tube-helix structure of Fig. 8 are similar to those of the tendril filament of Fig. 6, which in a large degree shows that the prediction of values of $C_{1}$ and $C_{2}$ are reasonable.

Figure 9 illustrates the investigated effects of the initial helical angle of a contained helix on the force-displacement relationship. For computation, the elastic modulus and Poisson's ratio of the cellulose fibril are taken as $0.6 \mathrm{GPa}$ and $0.4 \mathrm{GPa}$, respectively. The force-displacement curves for the different initial helical angles have been plotted. The helical angle refers to the angle between the axial direction and the tangential direction of the cellulose fibril. It is shown that under the same elongation, the tube-helix structure with a smaller initial helical angle needs a much larger tensile force than that with a relatively larger initial helical angle. This is because the relatively smaller initial helical angle can induce much greater superelasticity of the cellulose fibril helix.

These results indicate that the elasticity of the tube-helix structure is mainly due to the hyperelasticity of the tube and 


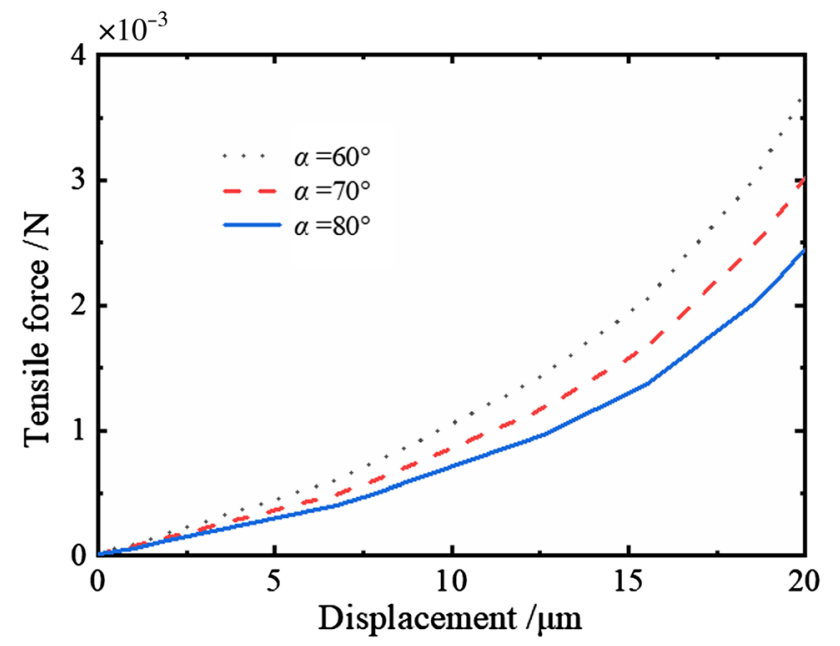

Fig. 9 Force-displacement curves of a tube-helix structure for different helical angles

superelasticity of the contained helix. And the latter plays a crucial role, especially during large elongation of the tendril filament. The tube-helix structure endows tendril cells with the ability to endure large elongation, which determines in a large degree the rubber-like elasticity of the tendril filament. It should be mentioned that during large elongation of the tendril filament, fracture of the tendril cell or fiber, and interactions among tendril cells, such as the friction and slipping, may occur, which can affect the mechanical behaviors of the tendril filament. Here, we mainly focus our attention on the elasticity of the hierarchical structure of the tendril, and the fracture and interactions of tendril cells are neglected for simplicity.

Based on the hierarchically chiral structures, the mechanical behavior of the tendril helix, filament and cell can be correlated to reveal the structure-property relationships. It is shown that through the combination of the rubber-like elasticity of the tendril filament manifested by its distinguished ability to endure large deformation and the macroscopic structural characteristic such as chirality perversion, the tendril helical structure can successfully fulfill the particular biological function of supporting the plant to climb, even in some extreme situations such as heavy wind and rain loads.

\section{Conclusions}

In this paper, the mechanical behaviors of the tendril helix, filament and cell are investigated via experiments and simulations. The results reveal that combination of the tendril helical structure with dual chirality or chiral perversion at a macroscale and the cellulose fibril helix at a subcellular level can provide enough elasticity for the biological needs of tendrils for supporting and climbing. The tendril filament can endure large elongation, exhibiting a rubber-like elasticity, which is derived mainly from the superelasticity of the cellulose fibril helix. This study not only sheds new insights into the structure-property relationship of climbing tendrils, but also paves the way for bioinspired design of fibrous composite systems with superior elasticity.

Acknowledgements This work was supported by the National Natural Science Foundation of China (Grants 11872273, 11472191, 11602163, and 11672297), the Major Program of the National Science Foundation of China (Grant 11890683), and the Opening Fund of State Key Laboratory of Nonlinear Mechanics. J.S. Wang thanks the support from the Australian Endeavour Research Fellowship.

\section{References}

1. Barthelat, F., Yin, Z., Buehler, M.J.: Structure and mechanics of interfaces in biological materials. Nat. Rev. Mater. 1, 16007 (2016)

2. Gao, H.J., Ji, B.H., Jäger, I.L., et al.: Materials become insensitive to flaws at nanoscale: lessons from nature. Proc. Natl. Acad. Sci. U.S.A. 100, 5597-5600 (2003)

3. Barthelat, F., Tang, H., Zavattieri, P.D., et al.: On the mechanics of mother-of-pearl: a key feature in the material hierarchical structure. J. Mech. Phys. Solids 55, 303-337 (2007)

4. Shao, Y., Zhao, H.P., Feng, X.Q., et al.: Discontinuous crackbridging model for fracture toughness analysis of nacre. J. Mech. Phys. Solids. 60, 1400-1419 (2012)

5. Monn, M.A., Weaver, J.C., Zhang, T.Y., et al.: New functional insights into the internal architecture of the laminated anchor spicules of Euplectella aspergillum. Proc. Natl. Acad. Sci. U.S.A. 112, 4976-4981 (2015)

6. Guo, Y., Chang, Z., Li, B., et al.: Functional gradient effects on the energy absorption of spider orb webs. Appl. Phys. Lett. 113, 103701 (2018)

7. Chen, Q., Shi, Q., Gorb, S.N., et al.: A multiscale study on the structural and mechanical properties of the luffa sponge from Luffa cylindrical plant. J. Biomech. 47, 1332-1339 (2014)

8. Fratzl, P., Weinkamer, R.: Nature's hierarchical materials. Prog. Mater Sci. 52, 1263-1334 (2007)

9. Zhao, Z.L., Zhao, H.P., Li, B.W., et al.: Biomechanical tactics of chiral growth in emergent aquatic macrophytes. Sci. Rep. 5, 12610 (2015)

10. Youngblood, J.P., Sottos, N.R.: Bioinspired materials for selfcleaning and self-healing. MRS Bull. 33, 732-737 (2008)

11. Gerbode, S.J., Puzey, J.R., McCormick, A.G., et al.: How the cucumber tendril coils and overwinds. Science 337, 1087-1091 (2012)

12. Isnard, S., Silk, W.K.: Moving with climbing plants from Charles Darwin's time into the 21st century. Am. J. Bot. 96, 1205-1221 (2009)

13. Wang, J.S., Wang, G., Feng, X.Q., et al.: Hierarchical chirality transfer in the growth of towel gourd tendrils. Sci. Rep. 3, 3102 (2013)

14. Goriely, A., Tabor, M.: Spontaneous helix hand reversal and tendril perversion in climbing plants. Phys. Rev. Lett. 80, 1564-1567 (1998) 
15. Darwin, C.: On the movements and habits of climbing plants. Bot. J. Linn. Soc. 9, 1-118 (1865)

16. Pieranski, P., Baranska, J., Skjeltorp, A.: Tendril perversion-a physical implication of the topological conservation law. Eur. J. Phys. 25, 613-621 (2004)

17. Wang, J.S., Cui, Y.H., Shimada, T., et al.: Unusual winding of helices under tension. Appl. Phys. Lett. 105, 043702 (2014)

18. Guo, Q., Dai, E., Han, X., et al.: Fast nastic motion of plants and bio-inspired structures. J. R. Soc. Interface 12, 0598 (2015)

19. Wan, G.C., Jin, C.R., Trase, I., et al.: Helical structures mimicking chiral seedpod opening and tendril coiling. Sensors. 18, 2973 (2018)

20. Wu, W.W., Cheng, L.C., Niu, Y.H., et al.: Compression twist deformation of novel tetrachiral architected cylindrical tube inspired by towel gourd tendrils. Extreme Mech. Lett. 20, 104111 (2018)

21. Chen, P.N., Xu, Y.F., He, S.S., et al.: Hierarchically arranged helical fiber actuators driven by solvents and vapours. Nat. Nanotechnol. 10, 1077-1083 (2015)
22. Deng, J., Xu, Y.F., He, S.S., et al.: Preparation of biomimetic hierarchically helical fiber actuators from carbon nanotubes. Nat. Protoc. 12, 1349-1358 (2017)

23. Chen, Y., Wang, R.R., Chan, K.H., et al.: A biomimetic conductive tendril for ultrastretchable and integratable electronics, muscles, and sensors. ACS Nano 12, 3898-3907 (2018)

24. Zhao, Z.L., Zhao, H.P., Cheng, Z., et al.: Analysis of bending and buckling of pre-twisted beams: a bioinspired study. Acta Mech. Sinica 30, 507-515 (2014)

25. Ma, T.Y., Wang, Y.N., Yuan, L., et al.: Timoshenko beam model for chiral materials. Acta Mech. Sinica 34, 549-560 (2018)

26. Hibbitt, D., Karlsson, B., Sorenser, P.: ABAQUS Theoretical Manual. Hibbitt, Karlsson \& Sorensen Inc., USA (1997)

27. Gent, A.N.: A new constitutive relation for rubber. Rubber Chem. Technol. 69, 59-61 (1996)

28. Ogden, R.W.: Non-linear elastic deformations. Eng. Anal. 1, 119 (1984) 